\title{
Delineation of electrocardiogram morphologies by using discrete wavelet transforms
}

\author{
Annisa Darmawahyuni, Siti Nurmaini, Hanif Habibie Supriansyah, Muhammad Irham Rizki Fauzi, \\ Muhammad Naufal Rachmatullah, Firdaus Firdaus, Bambang Tutuko \\ Intelligent System Research Group, Universitas Sriwijaya, Palembang 30139, Indonesia
}

\begin{tabular}{|c|c|}
\hline Article Info & ABSTRACT \\
\hline Article history: & \multirow{9}{*}{$\begin{array}{l}\text { The accuracy of electrocardiogram (ECG) delineation can affect the precise } \\
\text { diagnose for cardiac disorders interpretation. Some nonideal ECG presentation } \\
\text { can make a false decision in precision medicine. Besides, the physiological } \\
\text { variation of heart rate and different characteristics of the different ECG waves } \\
\text { in terms of shape, frequency, amplitude, and duration is also affected. This } \\
\text { paper proposes a discrete wavelet transform (DWT), non-stationary signal } \\
\text { analysis for noise removal, and onset-offset of PQRST feature extraction. A } \\
\text { well-known database from Physionet: QT database (QTDB) is used to validate } \\
\text { the DWT function for detecting the onset and offset of P-wave, QRS-complex, } \\
\text { and T-wave localization. From the results, P-peak detection gets the highest } \\
\text { result that achieves } 2.19 \text { and } 13.62 \text { milliseconds of mean error and standard } \\
\text { deviation, respectively. In contrast, Toff has obtained the highest error value } \\
\text { due to differences in the T-wave morphology. It can be affected by inverted or } \\
\text { biphasic T-waves and others. }\end{array}$} \\
\hline Received Mar 1, 2020 & \\
\hline Revised Nov 10, 2020 & \\
\hline Accepted Dec 5, 2020 & \\
\hline Keywords: & \\
\hline Delineation & \\
\hline Discrete wavelet transform & \\
\hline Electrocardiogram & \\
\hline Noise removal & \\
\hline
\end{tabular}

This is an open access article under the CC BY-SA license.

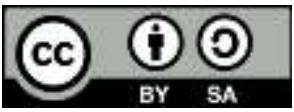

Corresponding Author:

Siti Nurmaini

Intelligent System Research Group

Faculty of Computer Science, Universitas Sriwijaya

Palembang 30139, Indonesia

Email: siti_nurmaini@unsri.ac.id, sitinurmaini@gmail.com

\section{INTRODUCTION}

A cardiologist analyzes the electrical function of the cardiac via electrocardiogram (ECG). Analysis of critical segments of ECG is a crucial thing for diagnosing cardiac disorders. However, in some cases, an ECG-based diagnosis can be difficult [1]. For example, the diagnosis of myocardial infarction (MI), one of coronary heart disease due to a lack of oxygen demand in the cardiac muscle tissue [2, 3]. The ECG form changes in ST-elevation, T-waveform, and the ST interval length. In other cases, the detection of QT and RR-interval for calculating QT-corrected (QTc) to inform long QT syndrome (LQTS), and P-wave abnormality to diagnose Atrial Fibrillation, are difficult due to the lack of symptoms [4-6]. A cardiologist needs to analyze ECG recordings that are acquired over several hours or even days, making the task very troublesome and time-consuming [1, 7,8]. Advanced computing systems can reduce such limitations permit the automatic interpretation of ECG. Precise ECG is essential in getting maximum benefits to interpret ECG recordings properly. The additional features of ECG need to be analyzed by the morphology of the different waves within the signal. The computing algorithms require specific points within the ECG wave segmentation, include the start (onset) to end (offset) of the QRS-complex, P, and T-waves.

Automatic segmentation with a low complexity algorithm of the ECG is challenging due to the Pwave's reduced amplitude, the high variability in the shape of the QRS-complex. The smooth transitions of the beginning and end of T-wave [9-11] The previous conventional algorithm is applied for automatic ECG 
segmentation in many works of literature, such as the hidden Markov model [12], multiple higher-order moments (MHOM) metric [13], continuous wavelet transform [14] and a bandpass Butterworth filter and the first derivative [15]. Laguna et al. [16] first proposed a very successful ECG segmentation approach based on second-order bandpass filtering the ECG and then differentiating it. In the end, different waves would be detected based on their zero-crossings and finding the nearest points exceeding empirical thresholds. Then, Li et al. also implemented a discrete wavelet transform (DWT) for detecting ECG characteristic points [17]. Bazomenos et al. [18] employed DWT with the simplest wavelet function, Haar function, for the extraction of the fiducial points from the ECG. The aforementioned techniques such as an ideal algorithm to be efficient from a computational perspective in ECG signal processing tasks.

This paper concerns developing a low-complexity algorithm for ECG delineation in PQRST detection. Wavelet transform (WT) function can be proposed on this approach because of its time-scale analysis nature [18]. Wavelet transform can be a promising function for time-frequency analysis. Besides, the wavelet transform can be characterized by the local regularity of signals. Another advantage of the wavelet transform is that it has limited energy because it is localized in time, allowing time and frequency analysis. Wavelet transform is divided into continuous wavelet transform (CWT) and discrete wavelet transform (DWT). However, in this paper, DWT is proposed for ECG signal denoising and feature extraction. DWT is an implementation of WT, which is promising in biomedical signal processing.

\section{RESEARCH METHOD}

This study experimented on the well-known physionet: QT database (QTDB), in which the training and testing set was generated. From the dataset, ECG raw has denoised by discrete wavelet transform (DWT), in which input signals are decomposed into wavelet levels and transferred to other layers as sequences. Then, a new input layer with a wavelet basis was designed for detection of start to the end P-wave, QRS-complex, and T-wave. The research methodology can be presented in Figure 1.

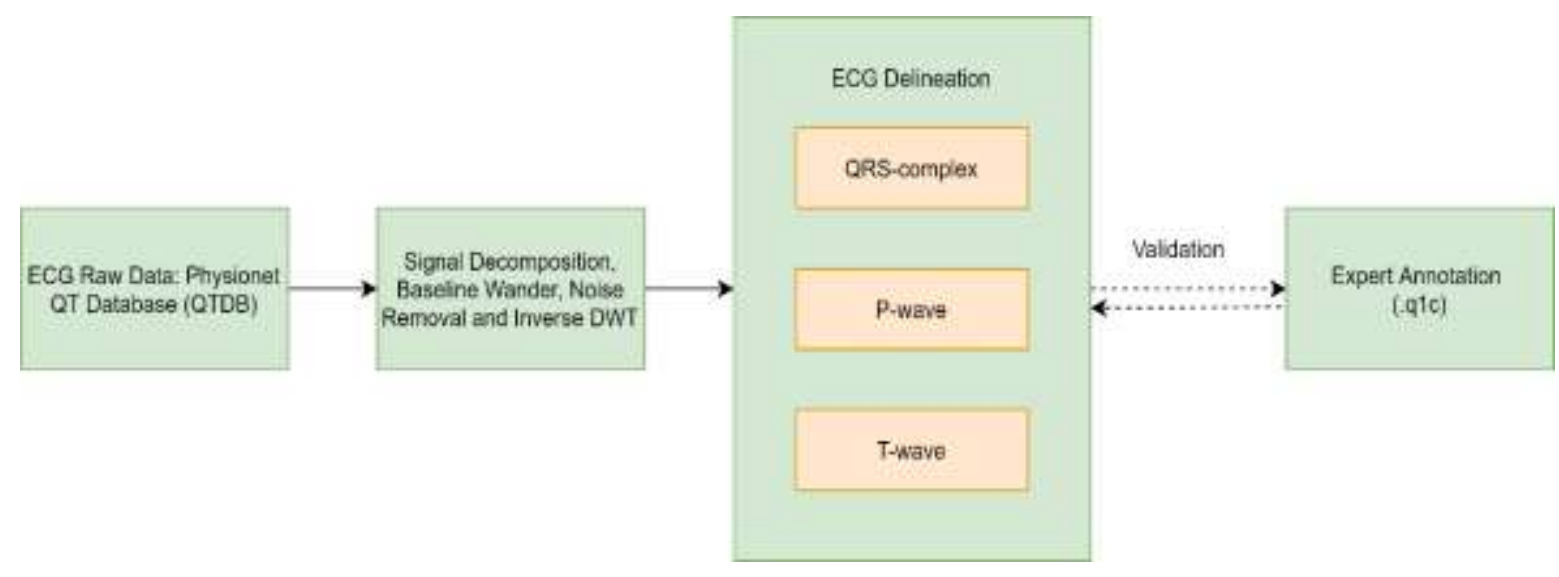

Figure 1. The workflow of ECG delineation

\subsection{Discrete wavelet transform}

Explaining wavelet transform (WT) is efficient when evaluating non-stationary signals such as ECG. WT can overcome the drawbacks of the fourier transform (FT) that only fits in stationary signals and able to analyze signals in the frequency domain. From computation complexity, WT is lower than FT, it was developed for signal compression and noise reduction [19]. WT uses a mother wavelet as a signal to be convoluted along with the original signal. Another advantage of WT is energy-efficient because it localized in time, which allows analysis of time and frequency [20]. In WT, there are also mother wavelets such as Haar, Daubechies, Biorthogonal, Coiflets, Symlets and Morlet. Then there is no absolute reason to apply a particular mother wavelet. The choice of the mother wavelet itself depends on the type of signal to be analyzed and applied. For ECG noise removal, there are two thresholding algorithms which applied when using wavelets popularized by Donoho and Johnstone [19], hard thresholding in (1) and soft thresholding in (2): 


$$
\begin{aligned}
& c \widehat{D}_{j}=\left\{\begin{array}{c}
c D_{j},\left|c D_{j}\right| \geq t \\
0,\left|c D_{j}\right| \leq t
\end{array}\right. \\
& c \widehat{D}_{j}=\left\{\begin{array}{c}
\operatorname{sign}\left(c D_{j}\right)\left(\left|c D_{j}\right|-t\right),\left|c D_{j}\right| \geq t \\
0,\left|c D_{j}\right| \leq t
\end{array}\right.
\end{aligned}
$$

where $c \widehat{D}_{j}$ and $c D_{j}$ is the wavelet coefficient after and before the thresholding process. ECG signals will be an input for wavelets decomposed according to the specified level (8 levels). The decomposition process is a downsampling process that produces a coefficient with a particular frequency at each level represented in Figure 2. The signal frequency will be divided by two in DWT because it passes through the high pass and low pass filter. Frequencies that pass through the high pass filter will enter the detail coefficient. Then the low pass filter will enter the approximation coefficient. DWT uses a series of scales and translations by following specific rules. The general rule used in DWT is 2 to the power of $n$, where $n$ is an integer [21]. Wavelets in DWT work as bank filters that decompose the signal into subband and look like tree structures [21]. The features can distinguish ECG from electrode contact noise, baseline drift, and power line interference. An algorithm based on wavelet transform is proposed for detecting the start to end of QRScomplex, $\mathrm{P}$ and T-waves.

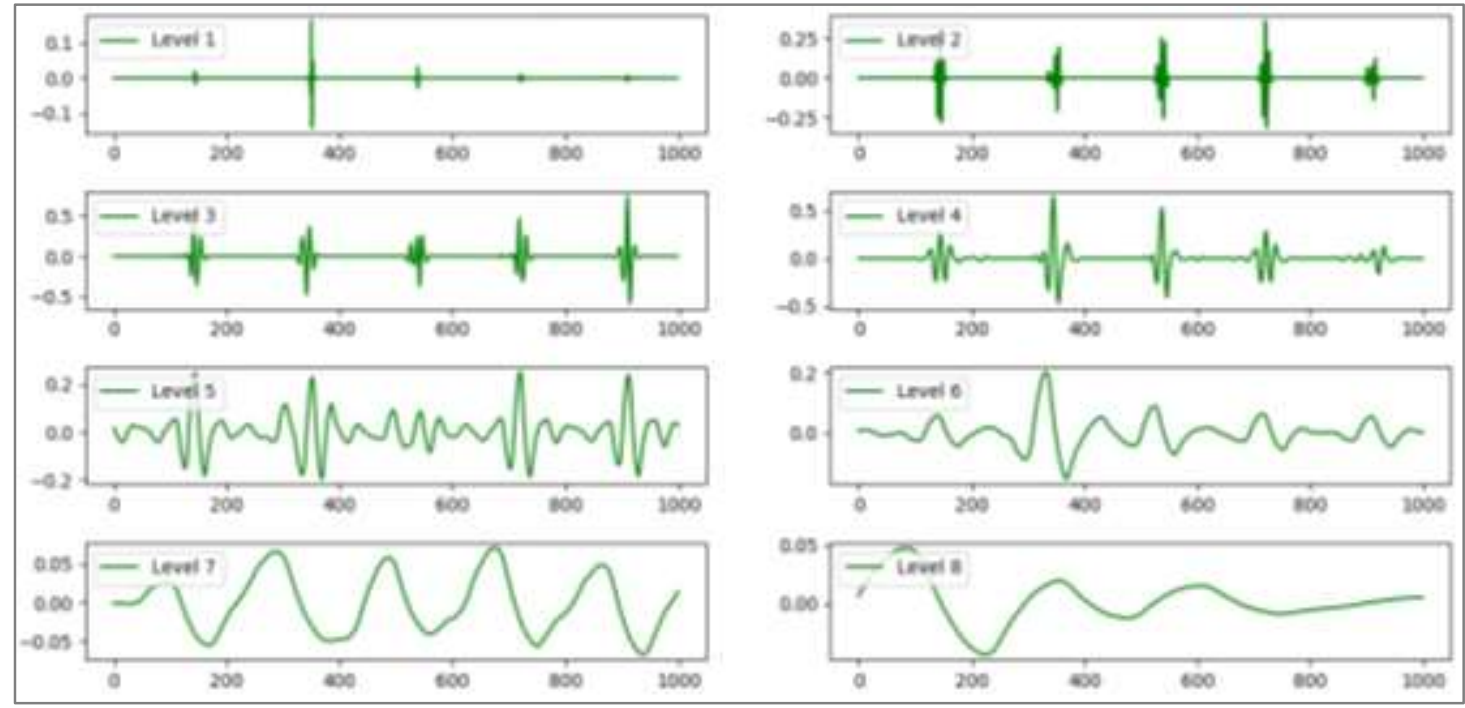

Figure 2. DWT results of ECG reconstruction 8 levels

\subsection{Data preparation}

ECG raw data is obtained from the Physionet public dataset's open-access: The QT Database (QTDB) [22]. The database consists of $105 \mathrm{ECG}$ records taken in 15 minutes with a sampling frequency of $250 \mathrm{~Hz}$ from two-channel Holter ECG recordings. This database consists of 15 records of MIT-BIH Arrhythmia, six records of MIT-BIH ST Change, 13 records of MIT-BIH Supraventricular Arrhythmia, 33 records of European ST-T, 24 records of sudden death patients from BIH, four records of MIT-BIH LongTerm ECG, and ten records of MIT-BIH Normal Sinus Rhythm. For this study, the records of sudden death are excluded. This database provides the input to the WFDB function ecgpuwave(), which gives us the exact position of all the $\mathrm{P}, \mathrm{R}$, and T-peaks found in the signal. The output of the ecgpuwave is written as a standard WFDB-format annotation file associated with the specified annotator. It is utilized as "ground truth" or label for the proposed ECG segmentation algorithm. Otherwise, only a complete waveform pattern of P-wave, QRS-complex, and T-wave is utilized in this study. Points of interest within the ECG include the $\mathrm{P}_{\text {on }}-\mathrm{P}_{\text {off }}$, $\mathrm{P}_{\text {off }}-\mathrm{QRS}_{\text {on }}, \mathrm{QRS}_{\text {on }}-\mathrm{R}_{\text {peak }}, \mathrm{R}_{\text {peak }}-\mathrm{QRS} \mathrm{S}_{\text {off }}, \mathrm{QRS}_{\text {off }}-\mathrm{T}_{\text {on }}$, and $\mathrm{T}_{\text {on }}-\mathrm{T}_{\text {off }}$ as shown in Figure 3. 


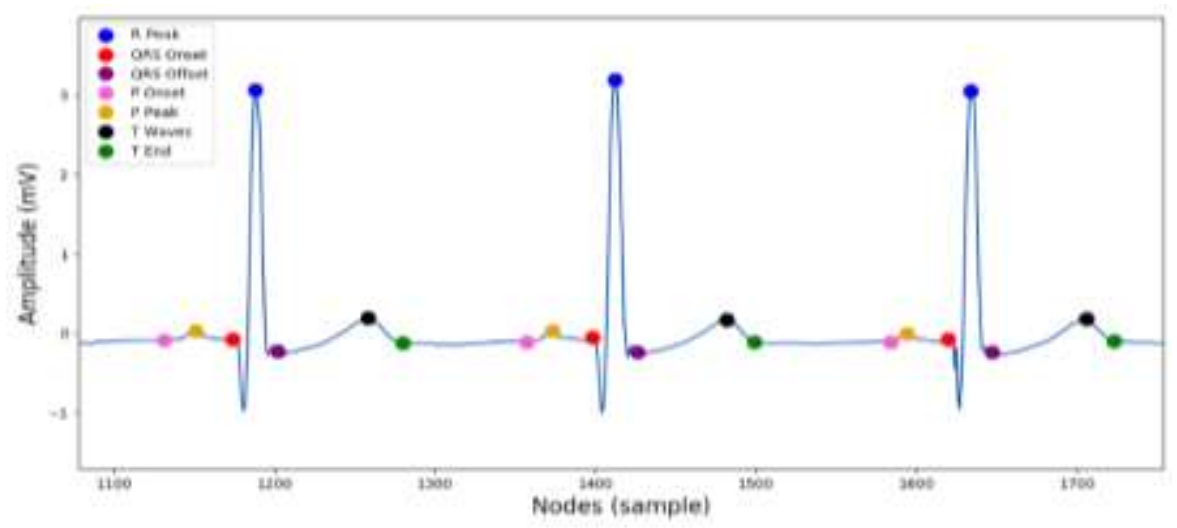

Figure 3. ECG onset-offset morphology

\subsection{ECG noise removal}

To analyze ECG signals, a clean ECG signal from noise is needed. DWT is used because it works as a bank filter that decomposite into several levels consisting of certain frequencies. The signal decomposition process is a signal downsampling process, whose function is to conduct frequency analysis at each level. The frequency will be accommodated on several coefficients according to the number of levels. After analysis of the coefficient of decomposition results, an upsampling or reconstruction coefficient will be carried out so that it produces a new signal. After the signal decomposition process, the baseline wander is removed by listing the level 8 approximation coefficient to 0 . Signals with baseline wanders do not have consistency in amplitude, because the midpoint of the signal is not at point 0 . Signals that have been processed by removing the baseline wander will be at the midpoint of 0 . The comparison of signal with baseline wander can be presented in Figure 4. Last, there is still noise in the form of high-frequency signals from the electrode motion artifact (ema) and muscle artifact (ma). In the ECG signal, high-frequency noise can be seen in the form of a spike or some kind of serration along with the main signal. To eliminate noise from high frequencies, the decomposition results' coefficient will go through the thresholding stage by selecting the threshold using universal and soft thresholding methods. The result of ECG noise removal can be seen in Figure 4.

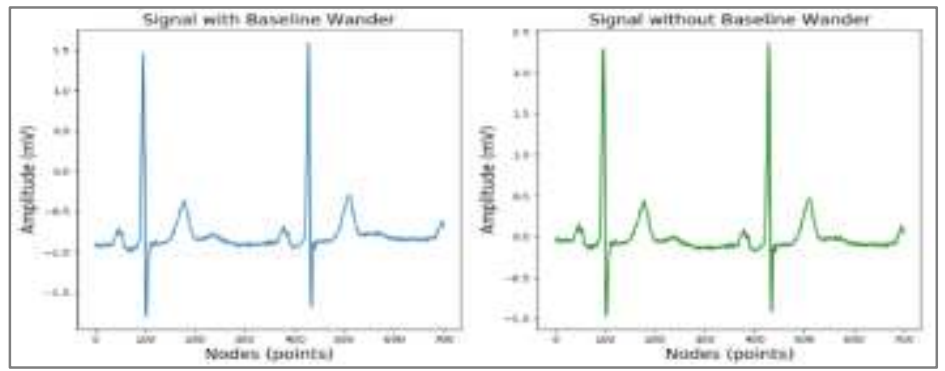

(a)

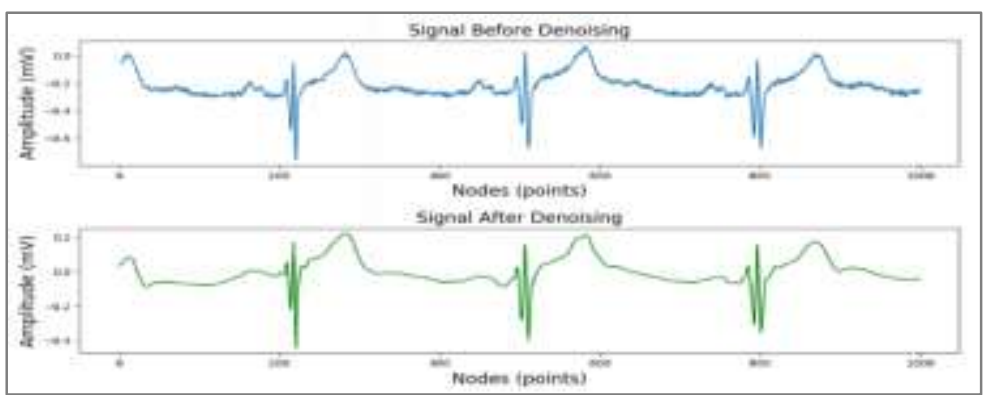

(b)

Figure 4. ECG noise removal pre-processing, (a) ECG baseline wander removal, (b) ECG noise removal 


\subsection{QRS-complex detection}

QRS-complex represents the most vital component for defining the condition of a human heart. However, to delineate R-peak in the QRS detector is not easy due to the non-stationary nature of ECG [23]. Initially, the QRS detector can be implemented by Pan and Thompkins algorithm [24], based on nonlinear transformations and the linear filtering techniques. Unfortunately, such an algorithm has a limitation of tracking time-varying ECG signal morphology and made detection of QRS-complex inaccurate. To overcome the problems, the method based on joint time-frequency analysis tools has been addressed, i.e. wavelet transforms [19, 23]. In previous [19], Lin et. al. proposed DWT and soft thresholding for ECG noise removal and feature detection, respectively. Bajaj et al. [23] implemented Stockwell transform that accurately identifies the QRS-complex of unclassifiable beats. From the results, the wavelet transform algorithm has the capability to QRS detector. Based on the results of 8 levels signal reconstruction in Figure 2 above, for the QRS detector, only decomposition level 2 (DL2), 3 (DL3), and 4 (DL4) is proposed. Those three decomposition levels show the similarity of QRS-complex morphology [19]. For R-peak detection, the combination of all signal values (positive or negative) is used as absolute values (abs DL234). Then, the crossing window of 0.5 seconds has been implemented to look for the maximum value on the abs DL234. If the maximum value is between $0.15-0.35$ seconds in the search window, the value is captured as a temporary $\mathrm{R}$-value $\left(t e m p \_R\right)$. Trace the search window by 0.1 seconds until the end of the signal. Find the maximum value on the abs DL234 signal with the search window of 5 seconds. Each maximum value obtained will be inserted into a temporary threshold list (temp_thr). Each value in temp_thr will be used in the following (3) to find the value of threshold 1 ;

$$
\text { threshold } 1=0.3 * \text { temp_thr }[i]
$$

In the next process, compare temp_R value with the threshold 1 . If the value of temp_R is greater than threshold 1, then the value is taken as the R-peak. Do a last check of the obtained R-peak with a search window width of 0.16 seconds ( 0.08 for each backward and forward). If the absolute value of the original signal is greater than the value of R-peak, then the value of R-peak is replaced by the absolute value of the original signal.

Furthermore, $\mathrm{QRS}_{\text {on }}$ has been detected by the R-peak location. If the amplitude value of R-peak is negative, get the maximum value in signal DL234 as Q-peak. While, if the amplitude value of R-peak is positive (point of R-peak is above), get the minimum value in signal DL234 as Q-peak. Then, find the zerocrossing point between two signals; denoised and DL234 reconstruction signal. The zero-crossing point is a condition where the point of the denoised signal intersects with the point of the DL234 reconstruction signal on the $\mathrm{y}$-axis $=0$. If there is a zero-crossing point, then capture it as $\mathrm{QRS}_{\mathrm{on}}$. If there is no zero-crossing point, find the maximum or minimum value in $0.03 \mathrm{~s}$ that represents the $\mathrm{QRS}_{\mathrm{on}}$. For $\mathrm{QRS}_{\text {off }}$ detection, the process is

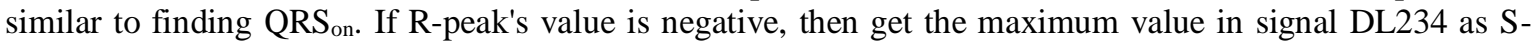
peak and find zero crossing point from S-peak to 0.1 seconds forward. Then, if there is a zero-crossing point, capture it as $\mathrm{QRS}_{\text {off }}$, or find the minimum value in signal DL234 as $\mathrm{QRS}_{\text {off }}$ while there is no zero-crossing point. There is a condition where the value of $\mathrm{R}$-peak is positive. Hence, get the minimum value in signal DL234 as S-peak and compare it with the threshold. If S-peak is greater than the threshold, then capture

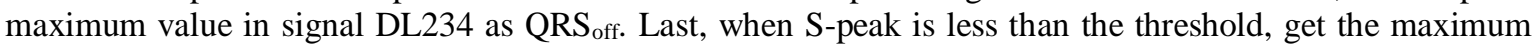
value in signal DL3 as QRSoff. The definition of the threshold can be calculated by,

$$
\operatorname{thr}(i)=0.4 \times \operatorname{Rpeak}(i)(4)
$$

\subsection{P-wave Detection}

P-wave is the first deflection of ECG that reflects the depolarization of the atrial muscle cells. It does not represent the contraction of that muscle, nor does it represent the firing of the SA node [25]. P-wave appears before the QRS-complex or the left side of the $\mathrm{QRS}_{\text {on. }}$ For the $\mathrm{P}$-wave delineation flowchart algorithm, decomposition signal level DL4 and DL5 (DL45) are used. An adaptive window is used for Ppeak detection. An original searching window (OSW) has proposed before by [19]. The (5) of OSW can be explained by,

$$
O S W=\left[Q R S_{\text {on }}-0.33 \times R R_{\text {mean } 20} \times f S: Q R S_{\text {on }}-15\right]
$$

where $R R_{\text {mean } 20}$ is average of the first twenty values of average $\mathrm{RR}$ and $f s$ is frequency sampling. From OSW, find the maximum value in DL45 (Pmax45) and the denoised signal (Pmax) on OSW. Then, calculate the threshold $\mathrm{P}\left(t h r_{P}\right)$ which defined as, 


$$
t h r_{P}=0.125 \times \max \left(\mid t h r_{D L 4+D L 5}[n]\right)
$$

There is a condition for P-peak detector from (6) if Pmax and Pmax45 are greater than $t h r_{P}$ then calculate the difference between Pmax and Pmax45. Then, get the maximum value in the denoised signal as P-peak if Pmax and Pmax45 is less than $t h r_{P}$. In addition, there is a condition that the difference in Pmax and Pmax45 is less than five points, so get the maximum value in denoised signal as P-peak, if not then capture the maximum value in signal DL45 as P-peak. Nevertheless, if the time difference between the maximum value noticed with the OSW and $\mathrm{QRS}_{\mathrm{on}}$ is too small, the searching window should be modified. Thus, the ration of $E p s_{P}$ is defined as,

$$
E p S_{P}=\frac{\text { the difference between the max value and } Q R S o n}{R R_{a v}}
$$

If $\operatorname{Eps}_{P}$ is less than 0.12 seconds, make a new windows size called Narrow Searching Windows (NSW), then capture the maximum value in denoised signal as P-peak. Furthermore, if the position of P-peak is greater than 25 points from $\mathrm{QRS}_{\text {on }}$ then change left OSW to $\left(\mathrm{QRS}_{\text {on }}-45\right)$ points. Obtain the maximum value in denoised signal on the new OSW as P-peak. The NSW can be defined as,

$$
\left.N S W=Q R S_{\text {on }}-0.18 \times R R_{\text {mean } 20} \times f s: Q R S_{\text {on }}-0.12 \times R R_{\text {mean } 20} \times f s\right]
$$

After the P-peak is detected in ECG delineation, take the position of P-peak and the left margin of OSW for searching P-peak. Then, get the minimum value in signal DL56 as Pon. If the difference of P-peak and Pon is greater than 20 points, then change the left margin of OSW to (P-peak - 20 points), capture the minimum value in signal DL56 as Pon.

\subsection{T-wave detection}

T-wave on ECG represents typically ventricular repolarization. It can characterize variations of normal cardiac electrophysiology or signs pathology [26]. Many potential presentations that cause T-wave abnormalities are associated with high morbidity and mortality without emergent intervention. When the ventricular muscles begin to relax, T-wave shows the repolarization of the ventricles. If T-wave shows negative morphology, it can indicate that the patient has heart ischemia. For detecting the $\mathrm{T}$ wave, the first create a searching window, searching windows T (SWT) with a window size (9):

$$
S W T=\left[Q R S_{\text {end }}+f S \times 0.1: Q R S_{\text {on }}+0.46 \times \sqrt{R R_{\text {mean } 20} \times f S}\right.
$$

Then, to determine the maximum absolute value in the noise removal results on the SWT or TMAX, need to define the limit of the amplitude of the T point, so that the threshold $\left(\gamma_{T}\right)$ is made (10):

$$
\gamma_{T}=0.125 \times \max \left(\left|y_{D L 4+D L 5}[n]\right|\right), n \in S W T
$$

where the value of $n$ is 20 samples before the main wave and 40 samples after the main wave. Furthermore, the amplitude value of TMAX is obtained. If the amplitude value of TMAX is positive, then take the maximum value on the DL45 reconstruction signal as T-peak. However, if the TMAX amplitude value is negative, then take the maximum absolute value on the reconstruction signal DL45 as a T-peak point. The value of the T-peak point must be more than the threshold value. If the value of the T-peak point when the TMAX is negative is below the threshold value, then repeat the step from the beginning with the reconstruction signal DL56.

\section{RESULTS AND ANALYSIS}

This study proposes symlet as mother function, sym5, for noise denoising and feature extraction. From the DWT reconstruction of 8 levels, the level of 2, 3, 4 is used for QRS-complex detection, then the level of 5 and 6, and level 4 and 5 for P-wave and T-wave detection, respectively. Based on DWT results, the result of onset-offset PQRST localization is compared to expert annotation (.q1c). Furthermore, to analyze the comparison of the DWT algorithm and annotation, the average errors and standard deviation is used, listed in Tables 1 and 2, respectively. Table 1 shows the mean error in all datasets of QTDB. From the results, the R-peak localization can be detected with minimum error in all datasets. This is also similar for P-wave detection, still, the result for specific MIT-BIH Long Term ECG is not good enough. However, for T-wave detection, all results are poor. The point gap between the DWT algorithm and annotation is hugely 
significant. It can be seen $\mathrm{n}$ in MIT-BIH Arrhythmia and ST Change datasets, the difference obtains 13.30 points and 13.41 points to the left (minus sign), respectively. The localization of T-wave is a difficult task, due to the variance of T-wave morphologies, such as inverted, only upwards, only downwards, biphasic negative-positive, and biphasic positive-negative. Overall, for all onset-offset PQRST detection, the best result of MIT-BIH Normal Sinus Rhythm is obtained. It is not surprising due to the morphology of ECG normal is easier to recognize.

The standard deviation result of ECG delineation can be listed in Table 2. As seen in Table 2, the Twave value gets a high deviation value. A greater deviation value presents the data points that are far from the average value (mean). It can be concluded that $\mathrm{T}$-peak or $\mathrm{T}_{\text {off }}$ detection is still must be explored. The misinterpretation of $\mathrm{T}_{\text {off }}$ localization can be affected the decision making for specific cardiac disease, such as the calculation of QTc that need the precise $\mathrm{QRS}_{\text {on }}$, R-peak, and $\mathrm{T}_{\text {off }}$ for LQTS diagnosis.

Table 1. Mean Error of ECG Delineation for All Data

\begin{tabular}{|c|c|c|c|c|c|c|c|c|}
\hline Database & Results & Pon & P-peak & R-peak & QRSon & QRSoff & T-peak & Toff \\
\hline \multirow[t]{2}{*}{ MIT-BIH Arrythmia } & Mean (point) & 0.27 & 1.77 & -0.68 & -0.80 & 1.29 & -4.09 & -13.30 \\
\hline & Mean (milisecond) & 1.08 & 7.10 & -2.72 & -3.20 & 5.16 & -16.38 & -53.18 \\
\hline MIT-BIH ST Change & Mean (point) & -0.34 & -1.12 & -0.91 & -1.75 & 1.32 & -3.33 & -13.41 \\
\hline \multirow{2}{*}{ MIT-BIH Supraventricular Arrhythmia } & Mean (point) & 2.59 & 0.62 & -2.00 & -2.30 & 2.12 & -1.08 & -7.45 \\
\hline & Mean (milisecond) & 10.37 & 2.50 & -8.00 & -9.20 & 8.47 & -4.31 & -29.82 \\
\hline European ST-T & Mean (point) & -0.647 & -0.273 & -0.099 & -1.531 & 1.754 & -0.468 & -7.266 \\
\hline MIT-BIH Long-Term ECG & Mean (milisecond) & 19.65 & 21.00 & -4.70 & 1.35 & -6.13 & 27.94 & -20.30 \\
\hline \multirow[t]{2}{*}{ MIT-BIH Normal Sinus Rhythm } & Mean (point) & -1.42 & 0.89 & -1.53 & -2.46 & 0.50 & -4.93 & -5.95 \\
\hline & Mean (milisecond) & -5.69 & 3.55 & -6.12 & -9.84 & 1.99 & -19.73 & -23.8 \\
\hline \multirow{2}{*}{ All QTDB } & Mean (point) & 0.25 & 0.55 & -0.81 & -1.61 & 1.38 & -1.45 & -8.22 \\
\hline & Mean (milisecond) & 1.00 & 2.19 & -3.23 & -6.42 & 5.53 & -5.78 & -32.88 \\
\hline
\end{tabular}

Table 2. Standard Deviation of ECG Delineation for All Data

\begin{tabular}{|c|c|c|c|c|c|c|c|c|}
\hline Database & Results & Pon & P-peak & R-peak & QRSon & QRSoff & T-peak & Toff \\
\hline MIT-BIH Arrythmia & Mean (point) & 4.59 & 3.74 & 1.35 & 3.59 & 3.57 & 14.18 & 12.68 \\
\hline MIT-BIH ST Change & Mean (point) & 3.57 & 2.62 & 1.25 & 1.96 & 2.56 & 10.77 & 13.10 \\
\hline \multirow[t]{2}{*}{ MIT-BIH Supraventricular Arrhythmia } & Mean (point) & 6.58 & 3.84 & 1.15 & 2.10 & 2.40 & 13.44 & 11.17 \\
\hline & Mean (milisecond) & 26.30 & 15.35 & 4.61 & 8.41 & 9.58 & 53.77 & 44.69 \\
\hline European ST-T & Mean (point) & 6.006 & 3.609 & 1.052 & 2.208 & 2.543 & 9.835 & 8.930 \\
\hline MIT-BIH Long-Term ECG & Mean (milisecond) & 26.31 & 18.50 & 4.49 & 8.18 & 12.15 & 54.04 & 50.66 \\
\hline \multirow[t]{2}{*}{ MIT-BIH Normal Sinus Rhythm } & Mean (point) & 4.93 & 1.74 & 0.60 & 2.06 & 2.07 & 10.55 & 9.37 \\
\hline & Mean (milisecond) & 19.74 & 6.95 & 2.42 & 8.25 & 8.27 & 42.19 & 37.47 \\
\hline \multirow{2}{*}{ All QTDB } & Mean (point) & 5.64 & 3.40 & 1.07 & 2.33 & 2.62 & 11.39 & 10.35 \\
\hline & Mean (milisecond) & 22.57 & 13.62 & 4.27 & 9.32 & 10.47 & 45.57 & 41.42 \\
\hline
\end{tabular}

The results of this study have compared to the previous studies in the literature for ECG delineation in Table $3[16,19,27]$. Table 3 lists the benchmark result of the onset and offset that is used in QTDB. All units are millisecond (ms). From the previous studies, our results show better results in P-peak detection, 2.19 \pm 13.62. However, it still gets poor performance in $\mathrm{T}_{\text {off }},-32.38 \pm 41.42$. The detection of P-peak can be related to determine of $\mathrm{P}$-wave and PR-segment to interpret atrial fibrillation and myocardial infarction, respectively. The detection of $\mathrm{P}$-wave signal-averaged ECG can detect atrial conduction delay. Also, for myocardial infarction, it can be used for analyzing the position of PR-segment to J-point (QRS $\mathrm{off}_{\text {). }}$.

Table 3. The Benchmark Study of ECG Delineation with the Previous Literature

\begin{tabular}{cccccccc}
\hline Authors & Pon & P-peak & R-peak & QRSon & QRSoff & T-peak \\
\hline Laguna et al. [16] & $2.0 \pm 14.8$ & $3.6 \pm 13.2$ & N/R & $4.6 \pm 7.7$ & $0.8 \pm 8.7$ & $0.2 \pm 13.9$ & $-1.6 \pm 18.1$ \\
Lin et al. [19] & $14 \pm 13.3$ & $4.8 \pm 10.6$ & N/R & $-3.6 \pm 8.6$ & $-1.1 \pm 8.3$ & $-7.2 \pm 14.3$ & $13.5 \pm 27$ \\
Mart'inez et al. [27] & $0.9 \pm 11.5$ & $4.5 \pm 12.1$ & $-0.1 \pm 9.3$ & $-2.2 \pm 10.1$ & $4 \pm 9.8$ & $-2.4 \pm 12.5$ & $-0.9 \pm 17.1$ \\
This Study & $1 \pm 22.57$ & $2.19 \pm 13.62$ & $-3.23 \pm 4.27$ & $-6.42 \pm 9.32$ & $5.53 \pm 10.47$ & $-5.78 \pm 45.57$ & $-32.88 \pm 41.42$ \\
\hline
\end{tabular}




\section{CONCLUSION}

The precise ECG delineation is still a common issue for interpreting cardiac disease. Some nonideal effects usually affect experts for correct diagnoses, such as baseline wander, power-line noise, motion, and muscle artifact. DWT is efficient since analyzing non-stationary signals for noise removal and feature detection. In this paper, DWT is used for noise removal and PQRST onset and offset detection. From the experiment of this study validated in the well-known dataset, the highest result of feature segmentation is Ppeak that achieve 2.19 and 13.62 of mean error and standard deviation, respectively. Unfortunately, T-wave gets the poor performance due to the morphology of the T-wave itself is not always normal. It can be affected by inverted T-wave, biphasic T-waves, and others. For further research, it can be explored for the automated way feature representation using deep learning. The algorithm's improvement can be enhanced to detect some specific disease, such as QTc for LQTS.

\section{ACKNOWLEDGMENT}

This work was supported by Basic Research Grants (096/SP2H/LT/DRPM/2019) from the Ministry of Research and Technology, and Unggulan Profesi Grants 2020 from Universitas Sriwijaya Indonesia.

\section{REFERENCES}

[1] P. Sodmann, M. Vollmer, N. Nath, and L. Kaderali, "A convolutional neural network for ECG annotation as the basis for classification of cardiac rhythms," Physiol. Meas., vol. 39, no. 10, 2018, doi: 10.1088/1361-6579/aae304.

[2] A. Darmawahyuni, et al., "Deep Learning with a Recurrent Network Structure in the Sequence Modeling of Imbalanced Data for ECG-Rhythm Classifier," Algorithms, vol. 12, no. 6, p. 118, 2019, doi: 10.3390/a12060118.

[3] A. Darmawahyuni, "Coronary Heart Disease Interpretation Based on Deep Neural Network," Comput. Eng. Appl. $J .$, vol. 8, no. 1, 2019, doi: 10.18495/comengapp.v8i1.288.

[4] M. A. C. Gil, "A new, simpler and better correction formula for the QT interval," J. Am. Coll. Cardiol., vol. 61, no. 10 Supplement, p. E294, 2013.

[5] B. Vandenberk et al., "Which QT correction formulae to use for QT monitoring?," J. Am. Heart Assoc., vol. 5, no. 6, p. e003264, 2016, doi: 10.1161/JAHA.116.003264.

[6] N. Nuryani, et al., "RR-Interval variance of electrocardiogram for atrial fibrillation detection," in Journal of Physics: Conference Series, vol. 776, no. 1, 2016, doi: 10.1088/1742-6596/776/1/012105.

[7] S. Nurmaini et al., "Deep Learning-Based Stacked Denoising and Autoencoder for ECG Heartbeat Classification," Electronics, vol. 9, no. 1, p. 135, 2020, doi: 10.3390/electronics9010135.

[8] S. Nurmaini et al., "An Automated ECG Beat Classification System Using Deep Neural Networks with an Unsupervised Feature Extraction Technique,” Appl. Sci., vol. 9, no. 14, 2019, doi: 10.3390/app9142921,

[9] I. Beraza and I. Romero, "Comparative study of algorithms for ECG segmentation," Biomed. Signal Process. Control, vol. 34, pp. 166-173, 2017, doi: 10.1016/j.bspc.2017.01.013.

[10] S. Nurmaini, A. Gani, and others, "Cardiac Arrhythmias Classification Using Deep Neural Networks and Principle Component Analysis Algorithm.," Int. J. Adv. Soft Comput. Its Appl., vol. 10, no. 2, 2018.

[11] Siti Nurmaini, et al., "Deep Neural Networks Classifiers on The Electrocardiogram Signal for Intelligent Interpretation System," Sriwij. Int. Conf. Med. Sci., 2018.

[12] M. Akhbari, et al., "ECG segmentation and fiducial point extraction using multi hidden Markov model," Comput. Biol. Med., vol. 79, pp. 21-29, 2016, doi: 10.1016/j.compbiomed.2016.09.004.

[13] A. Ghaffari, M. R. Homaeinezhad, M. Khazraee, and M. M. Daevaeiha, "Segmentation of holter ECG waves via analysis of a discrete wavelet-derived multiple skewness--kurtosis based metric,” Ann. Biomed. Eng., vol. 38, no. 4, pp. 1497-1510, 2010, doi: 10.1007/s10439-010-9919-3.

[14] M. V/'litek, J. Hrubeš, and J. Kozumpll'lik, "A wavelet-based ECG delineation with improved P wave offset detection accuracy,” Anal. Biomed. Signals Images, vol. 20, pp. 160-165, 2010.

[15] C. R. Vázquez-Seisdedos, et al., "New approach for T-wave end detection on electrocardiogram: Performance in noisy conditions," Biomed. Eng. Online, vol. 10, no. 1, p. 77, 2011, doi: 10.1186/1475-925X-10-77.

[16] P. Laguna, R. Jané, and P. Caminal, "Automatic detection of wave boundaries in multilead ECG signals: Validation with the CSE database," Comput. Biomed. Res., vol. 27, no. 1, pp. 45-60, 1994, doi: 10.1006/cbmr.1994.1006.

[17] C. Li, C. Zheng, and C. Tai, "Detection of ECG characteristic points using wavelet transforms," IEEE Trans. Biomed. Eng., vol. 42, no. 1, pp. 21-28, 1995, doi: 10.1109/10.362922.

[18] E. B. Mazomenos et al., "A low-complexity ECG feature extraction algorithm for mobile healthcare applications," IEEE J. Biomed. Heal. informatics, vol. 17, no. 2, pp. 459-469, 2013, doi: 10.1109/TITB.2012.2231312.

[19] H.-Y. Lin, S.-Y. Liang, Y.-L. Ho, Y.-H. Lin, and H.-P. Ma, "Discrete-wavelet-transform-based noise removal and feature extraction for ECG signals," Irbm, vol. 35, no. 6, pp. 351-361, 2014, doi: 10.1016/j.irbm.2014.10.004.

[20] T. R. G. Nair, A. P. Geetha, and M. Asharani, "Adaptive wavelet based identification and extraction of PQRST combination in randomly stretching ECG sequence," in 2013 IEEE China Summit and International Conference on Signal and Information Processing, pp. 278-282, 2013, doi: 10.1109/ChinaSIP.2013.6625344.

[21] R. Haddadi, E. Abdelmounim, M. El Hanine, and A. Belaguid, "Discrete wavelet transform based algorithm for recognition of QRS complexes," in 2014 International Conference on Multimedia Computing and Systems 
(ICMCS), pp. 375-379, 2014, doi: 10.1109/ICMCS.2014.6911261.

[22] P. Laguna, R. G. Mark, A. Goldberg, and G. B. Moody, "A database for evaluation of algorithms for measurement of QT and other waveform intervals in the ECG," in Computers in cardiology 1997, pp. 673-676, 1997, doi: 10.1109/CIC.1997.648140.

[23] A. Bajaj and S. Kumar, "QRS complex detection using fractional Stockwell transform and fractional Stockwell Shannon energy," Biomed. Signal Process. Control, vol. 54, p. 101628, 2019, doi: 10.1016/j.bspc.2019.101628.

[24] J. Pan and W. J. Tompkins, "A real-time QRS detection algorithm," IEEE Trans. Biomed. Eng, vol. 32, no. 3, pp. 230-236, 1985, doi: 10.1109/TBME.1985.325532.

[25] D. E. Becker, "Fundamentals of electrocardiography interpretation," Anesth. Prog., vol. 53, no. 2, pp. 53-64, 2006, doi: 10.2344/0003-3006(2006)53[53:FOEI]2.0.CO;2.

[26] B. J. Kenny and K. N. Brown, "ECG T Wave," 2019.

[27] J. P. Mart'inez, R. Almeida, S. Olmos, A. P. Rocha, and P. Laguna, "A wavelet-based ECG delineator: evaluation on standard databases," IEEE Trans. Biomed. Eng., vol. 51, no. 4, pp. 570-581, 2004, doi: 10.1109/TBME.2003.821031. 